\title{
Vorwort.
}

Seine Entstehung verdankt das vorliegende Werk einerseits häufigen Anregungen mir befreundeter Fachgenossen, die nach dem Erscheinen meiner kleinen Einführung in die Maxwellsche Theorie es mir nahe legten, ein größeres Werk über das Gesamtgebiet der theoretischen Physik zu schreiben; anderseits war auch ich im Laufe meiner zehnjährigen akademischen Tätigkeit durch Unterredungen mit meinen Hörern zu der Überzeugung gelangt, daß ein Buch, das die theoretische Physik etwa in dem Umfange darstellt, wie sie in einem fünf- bis sechssemestrigen Vorlesungskursus bei vier Wochenstunden behandelt werden kann, nicht überflüssig sein dürfte und seinen Platz neben den vorhandenen Werken einnehmen könnte. Ich bin deshalb einer Aufforderung des Herrn Verlegers gerne gefolgt und lege heute den Fachgenossen den ersten Band vor. Er enthält die Mechanik diskreter materieller Punkte, starrer Körper und die Mechanik der Kontinua, d. h. Elastizität und Hydrodynamik. Darin ist die Akustik mitverarbeitet, die ja zum Teil der Punktmechanik, zum Teil der Elastizität oder Hydrodynamik angehört. Der zweite Band soll die Wärmelehre, die Elektrizität und die Optik in moderner Darstellung bringen.

Natürlich kann es sich bei einem solchen Buche im wesentlichen nur um eine Auswahl aus dem ungeheuren Stoffe handeln; da eine allgemeine Einigung darüber unmöglich ist, so kann ich nur hoffen, daß ich im allgemeinen eine akzeptable Mittellinie getroffen habe. Vielleicht darf ich noch hervorheben, daß ich mit besonderer Liebe die Schwingungsprobleme behandelt habe, die einmal zur Vorbereitung auf die elektrischen Wellen im zweiten Bande dienen sollen, und denen anderseits ein großer Teil meiner eigenen wissenschaftlichen Tätigkeit angehört. Hier bot sich auch Gelegenheit, z. B. bei den Saitenschwingungen, die Bedeutung der Integralgleichungen für das Problem der Entwicklung willkürlicher Funktionen nach Systemen vorgeschriebener zu zeigen; um dabei und bei ähnlichen Gelegenheiten den physikalischen Kern nicht mit rein 
mathematischen Untersuchungen zu belasten, habe ich die Anordnung so getroffen, daß diese Abschnitte beim ersten Studium überschlagen werden können. Überhaupt ist Kenntnis des Inhaltes des XIV. und XV. Kapitels (Schwingungen von Saiten und Membranen sowie Schwirgungen von Stäben und Platten) für das Folgende nicht notwendig; sie bilden vielmehr ein Gebiet für sich von wesentlich akustischem Interesse.

Ich möchte noch ein Wort sagen über den Gebrauch der Vektoranalysis. Die dieser angehörenden Begriffe und Formeln habe ich, da ich sie nicht als bekannt voraussetzen konnte, an den Stellen, an denen sie sich zwanglos darboten, entwickelt und dann im folgenden benutzt, ohne jedoch ausschließlich Gebrauch davon zu machen. Vielmehr habe ich in manchen Kapiteln die Koordinatendarstellung bevorzugt. Dies scheint mir in einem Lehrbuche geradezu notwendig zu sein, schon damit der Lernende die wichtigsten Formeln, z. B. die Gleichungen der Elastizität und Hydrodynamik, in beiderlei Gestalt kennen lernt; denn so braucht er sie beim Studium der Literatur.

Es ist kaum notwendig, zu sagen, daß ich bei meiner Darstellung die gesamte mir zugängliche Lehrbuchliteratur zu Rate gezogen habe. Ich möchte hier neben den Lehrbüchern der Hydrodynamik von Lamb und W. Wièn namentlich die Monographie über den Kreisel von Klein und Sommerfeld, sowie die Mechanik von $\mathrm{Hamel}$ als diejenigen Werke nennen, die mich in vieler Hinsicht beeinflußt haben. Ferner möchte ich noch hier hervorheben, daß ich durch häufige Bezugnahme auf das vortreffliche Tabellenwerk von Jahnke und Emde (Funktionentafeln mit Formeln und Kurven, Leipzig 1909) meine Darstellung entlasten konnte; ich habe um so unbedenklicher auf dieses Werk mich bezogen, als es in der Hand jedes Studierenden der Mathematik und Physik sein sollte.

Zahlreichen Fachgenossen habe ich für ihre liebenswürdige und stets bereite Hilfe zu danken. Vor allem Herrn Professor Dr. Adolf Kneser, mit dem ich nicht nur fast alle Partien des Buches besprechen konnte, sondern der mich mit seinem Rate ganz besonders bei Abfassung der Kapitel XIV und XV unterstützt hat, in denen die Integralgleichungen benutzt werden. Diese hatte er auch die Freundlichkeit, in den Korrekturbogen noch einmal durchzusehen. In den genannten Kapiteln habe ich übrigens ausgiebig die Anregungen verwertet, die ich in einer Vorlesung über die Theorie der Integralgleichungen von Kneser empfangen habe. Herr Professor Dr. Alexander Pflüger in Bonn hat eine ganze Korrektur 
gelesen und mir zahlreiche wertvolle Verbesserungsvorschläge, namentlich in pädagogischer Hinsicht, gemacht. Mein Kollege, Herr Privatdozent Dr. Walter Schnee, hat die Mechanik der materiellen Punkte und der starren Körper im Manuskript gelesen, viele Ungenauigkeiten berichtigt, die Darstellung in vielen Punkten rerbessert. Von ihm rührt ferner die elegante Darstcllung der Sätze über Transformation quadratischer Formen in Nummer 58 her. In gleicher Weise bin ich Herrn Privatdozenten Dr. Fritz Reiche in Berlin für Durchsicht des Manuskriptes der Mechanik der Kontinua verpflichtet, deren Darstellung er durch viele Ratschläge gefördert hat. Eine Schülerin von mir, Fräulein Di. Stallwitz, siellte mir ihre sorgfältig ausgearbeiteten Vorlesungshefte zur Verfügung, die ich als Grundlage für die erste Niederschrift des Manuskriptes benutzt habe. Herr Dr. Gustav Groß hatte die Freundlichkeit, eine ganze Korrektur zu lesen; Herr cand. phil. Paul Hahn hat mit großem Geschick die Figuren gezeichnet. Ihnen allen gebührt mein aufrichtiger Dank! In nicht geringerem Maße schulde ich diesen auch dem Herrn Verleger, der auf alle moine Wünsche in der bereitwilligsten Weise einging.

Möge der Erfolg die aufgewandte Mühe lohnen!

Breslau, Ostern 1914.

\section{Clemens Schaefer.}


